\title{
Inhibition of Fatty Acid Synthase (FASN) synergistically enhances the efficacy of 5-fluorouracil in breast carcinoma cells
}

\author{
ALEJANDO VAZQUEZ-MARTIN ${ }^{1,2,3}$, SANTIAGO ROPERO $^{4}$, JOAN BRUNET ${ }^{1,2,3}$, \\ RAMON COLOMER $^{1,2,3}$ and JAVIER A. MENENDEZ ${ }^{1,2,3}$ \\ ${ }^{1}$ Catalan Institute of Oncology (ICO)-Health Services Division of Catalonia; ${ }^{2}$ Girona Biomedical Research Institute (IdIBGi); \\ ${ }^{3}$ Medical Oncology, Dr Josep Trueta University Hospital of Girona, Girona, Catalonia; \\ ${ }^{4}$ Epigenetics Laboratory, Spanish National Cancer Centre (CNIO), Madrid, Spain
}

Received February 15, 2007; Accepted May 21, 2007

\begin{abstract}
The lipogenic enzyme fatty acid synthase (FASN) is differentially overexpressed and hyperactivated in a biologically aggressive subset of breast carcinomas and minimally in most normal adult tissues, rendering it an interesting target for anti-neoplastic therapy development. We previously reported that the FASN blockade can induce a synergistic chemosensitization of breast cancer cells to microtubule interfering agents (MIAs) such as docetaxel, paclitaxel and vinorelbine. Upon pharmacological inhibition of FASN activity using the natural antibiotic cerulenin [(2S,3R)-2,3-epoxy-4-oxo-7E,10E-dodecadienamide], we evaluated the role of FASN-catalyzed endogenous fatty acid biogenesis on the sensitivity of SK-Br3, MCF-7 and MDAMB-231 breast cancer cell lines to the anti-metabolite 5fluorouracil (5-FU). Cells were exposed simultaneously to cerulenin and 5-FU, sequentially to 5-FU followed by cerulenin or cerulenin followed by 5 -FU. Cell viability was determined by MTT assays and the increase in 5-FU-induced cell growth inhibition was measured by dividing $5-\mathrm{FU} \mathrm{IC}_{30}$ and $\mathrm{IC}_{50}$ values (i.e., $30 \%$ and $50 \%$ inhibitory concentrations, respectively) that were obtained in the absence of cerulenin by those in its presence. Co-exposure to cerulenin enhanced 5-FU efficacy up to 20-, 81-, and 58-times in SK-Br3, MCF-7 and MDA-MB-231 cells, respectively. Pre-treatment with cerulenin followed by the addition of 5-FU increased 5-FU efficacy up to 31-, 87-, and 126-times in SK-Br3, MCF-7 and MDA-MB-231 cells, respectively. Pre-treatment with 5-FU followed by the addition of cerulenin augmented 5-FU
\end{abstract}

Correspondence to: Dr Javier A. Menendez, Instituto Catalán de Oncología de Girona (ICO Girona), Fundación del Instituto de Investigación Biomédica de Girona, Dr Josep Trueta (IdIBGi), Medical Oncology, Hospital Universitario de Girona, Avenida de Francia s/n; 17007 Girona, Catalonia, Spain

E-mail: jmenendez@ico.scs.es

Key words: fatty acid synthase, 5-fluorouracil, chemotherapy, breast cancer efficacy up to 107-, 20-, and 18-times in SK-Br3, MCF-7 and MDA-MB-231 cells, respectively. When isobologram transformations of multiple dose-response analyses were performed to detect in vitro synergy, we concluded that the nature of the interaction between cerulenin and 5-FU in individual breast cancer cells lines generally exhibited sequence-dependency. Thus, while synergism was mainly observed when breast cancer cells were exposed to 5-FU prior to cerulenin, moderate synergism or additive interactions was obtained either when the chemical FASN blocker preceded 5-FU or when both drugs were concurrently administered. Of note, no antagonist interactions occurred upon any schedule of combined treatment with cerulenin and 5-FU. Our current findings revealing a schedule-dependent synergistic interaction between 5-FU and cerulenin represents, to the best of our knowledge, the first evidence that FASN-catalyzed de novo FA biogenesis plays a key role in regulating breast cancer cell response to antimetabolite-based therapies.

\section{Introduction}

The biosynthetic protein fatty acid synthase (FASN) is the major enzyme required for the anabolic conversion of dietary carbohydrates into fatty acids (FAs). In well-nourished individuals the anabolic-energy-storage role of FASN is of minor importance due to high levels of dietary fat and most normal cells and tissues under-utilize endogenous FAs. Indeed, most normal tissues, even those with a high cellular turnover, seem to preferentially use circulating FAs for the synthesis of structural lipids (1-3). Under normal physiological conditions, any FASN increase is tightly regulated by a number of environmental, hormonal and nutritional signals (4). A completely different picture emerges when evaluating the role of FASN-catalyzed endogenous FA biogenesis in tumor cells. After numerous clinical and basic research studies, it now appears that a biological subset of carcinomas express high levels of FASN protein and undergo significant endogenous FA biosynthesis independently of the regulatory signals downregulating FASN expression and FAs biosynthesis in normal cells (5). Importantly, FASN overexpression/hyperactivity particularly occurs in epithelial carcinomas with poor prognosis and serves as an independent prognostic indicator of adverse clinical outcome (6), supporting the notion that FASN 
is involved in the development, maintenance and enhancement of the malignant phenotype.

The fluoropyrimidine 5-fluorouracil (5-FU) is an anticancer drug widely used in the treatment of human tumours. 5-FU was developed in the 1950s following the observation that rat hepatomas used the pyrimidine uracil more rapidly than normal tissues, indicating that uracil metabolism was a potential target for antimetabolite therapy. Antimetabolite drugs such as 5-FU work by inhibiting essential biosynthetic processes, or by being incorporated into macromolecules, such as DNA and RNA, inhibiting their normal function. In this regard, the mechanism of cytotoxicity of 5-FU has been ascribed to the misincorporation of fluoronucleotides into RNA and DNA and to the inhibition of the nucleotide synthetic enzyme thymidilase synthase leading to DNA strand breaks and cell death (7). Over the past 20 years, an increased understanding of the mechanism of action has led to the development of strategies aimed at increasing the anticancer activity of 5-FU. Despite these advances, drug resistance remains a significant limitation to the clinical use of 5-FU.

Our group previously demonstrated that the inhibition of FASN induces a synergistic chemosensitization of human cancer cells to the anti-tubulinic agents docetaxel (Taxotere ${ }^{\mathrm{TM}}$ ), paclitaxel $\left(\right.$ Taxol $^{\mathrm{TM}}$ ) and vinorelbine (Navelbine $\left.{ }^{\mathrm{TM}}\right)(8-10)$. In addition, we have also reported that simultaneous targeting of FASN and HER2 by the chemical FASN inhibitor cerulenin and the anti-HER 2 humanized monoclonal antibody trastuzumab (Herceptin ${ }^{\mathrm{TM}}$ ), respectively, was synergistically cytotoxic in HER2-positive cancer cells (11). Similarly, concurrent RNAi-mediated silencing of FASN and HER2 genes synergistically stimulated apoptotic cell death in HER2 overexpressors (11). These findings, altogether, were pioneers at revealing that novel therapeutic strategies aimed to specifically suppress breast cancer-associated FASN hyperactivity might be successfully exploited to improve the efficacy of existing breast cancer therapies based on either microtubule-interfering agents (MIAs) or HER2-targeted antibodies. It remains to be addressed, however, whether tumor-associated FASN might also actively modulate the efficacy of other widely used anti-breast cancer agents with different mechanisms of action, including antimetabolites such as 5-FU. In this study, using a wide panel of human breast cancer-derived cell models naturally exhibiting graded levels of FASN, we have systematically assessed the nature of the interaction between a naturally occurring chemical FASN inhibitor (i.e., cerulenin) and 5-FU.

\section{Material and methods}

Cell culture. MDA-MB-231 and MCF-7 breast cancer cells were obtained from the American Type Culture Collection (ATCC) and they were routinely grown in Dulbecco's modified Eagle's medium (DMEM, Gibco) containing 10\% heat-inactivated foetal bovine serum (FBS, Bio-Whittaker), $1 \%$ L-glutamine, $1 \%$ sodium pyruvate, $50 \mu / \mathrm{ml}$ penicillin and $50 \mu \mathrm{g} / \mathrm{ml}$ streptomycin. SK-Br3 breast cancer cells were obtained from Dr. G. Marquez (Centro Nacional de Biotecnología, Madrid, Spain) and they were passaged in McCoy's 5 A medium containing $10 \%$ heat-inactivated foetal bovine serum, $1 \%$ l-glutamine, $1 \%$ sodium pyruvate, $50 \mu / \mathrm{ml}$ penicillin and $50 \mu \mathrm{g} / \mathrm{ml}$ streptomycin. Cells were maintained at $37^{\circ} \mathrm{C}$ in a humidified atmosphere of $95 \%$ air and $5 \% \mathrm{CO}_{2}$. Cells were in the logarithmic phase of growth at the time of the drug sensitivity assays.

Chemicals. 5-FU was purchased from Ferrer-Pharma, diluted in saline solution and stored as stock at $-20^{\circ} \mathrm{C}$. Cerulenin was obtained from Sigma-Aldrich, diluted in Dimethyl Sulfoxide (DMSO) as a stock solution $(50 \mathrm{mg} / \mathrm{ml})$ and stored in the dark at $-20^{\circ} \mathrm{C}$. For experimental use, $5-\mathrm{FU}$ and cerulenin were freshly prepared from stock solutions and diluted with a growth medium. Control cells were cultured in a medium containing the same concentration $(\mathrm{v} / \mathrm{v})$ as the experimental cultures with treatments. The vehicle solutions had no noticeable influence on the proliferation of experimental cells.

Chemosensitivity assays. Drug sensitivity was determined using a standard colorimetric MTT (3-4,5-dimethylthiazol-2-yl-2,5diphenil-tretazolium bromide) assay. Briefly, cells were plated out at a density of $10^{4}$ cells/100 $\mu 1 /$ well in 96-well microtiter plates and allowed an overnight period for attachment. Then the medium was removed and a fresh medium, along with various concentrations of cerulenin and 5-FU as single agents or combinations of compounds as specified, were added to the cultures in parallel. Agents were studied either concurrently or sequentially, with the first agent washed out prior to the introduction of the second drug. Control cells without agents were cultured using the same conditions with comparable media changes. Following treatment, cells were fed with a drug-free medium (100 $\mu \mathrm{l} /$ well) and MTT (10 $\mu \mathrm{l} /$ well, $5 \mathrm{mg}$ per $\mathrm{ml}$ in PBS) and incubation was prolonged for $3 \mathrm{~h}$ at $37^{\circ} \mathrm{C}$. After removing the supernatants, the MTT-formazan crystals were dissolved in DMSO (100 $\mu \mathrm{l} /$ well) and the absorbance was measured at $570 \mathrm{~nm}$ in a multi-well plate reader (Model Anthos Labtec 20101.7 reader).

The growth inhibitory effects from exposure of cells to each compound alone and their combination for a particular schedule were analyzed generating concentration-effect curves as a plot of the fraction of unaffected (surviving) cells versus drug concentration. Growth inhibition was expressed as a percentage of the untreated controls that were processed simultaneously, using the following equation: $\left(\mathrm{A}_{570}\right.$ of treated sample/ $\mathrm{A}_{570}$ of untreated sample) x100. The $\mathrm{IC}_{30}$ (i.e., cytostatic condition) and $\mathrm{IC}_{50}$ (i.e., cytotoxic condition) values were defined as the drug concentrations inhibiting cell growth by either 30 or $50 \%$ (i.e., 30 or $50 \%$ reduction of absorbance at $570 \mathrm{~nm}$ ), compared with untreated controls, respectively. It was established that optical density was directly proportional to the cell number up to the density reached by the end of the assay. The degree of sensitization to 5-FU by cerulenin was determined by dividing both the $\mathrm{IC}_{30}$ and $\mathrm{IC}_{50}$ values of the control cells by those obtained when cells were exposed to cerulenin before, during or after the exposure to 5-FU.

Data analysis for combination treatment. The interaction between cerulenin and 5-FU was evaluated by the isobologram technique, a dose-oriented geometric method that is widely used to assess the interaction between two antitumor drugs or between drugs and radiotherapy (12). The mathematical 
Table I. Effects of cerulenin-induced inhibition of FASN activity on the sensitivity of SK-Br3 cells to 5-FU.

\begin{tabular}{|c|c|c|c|c|c|}
\hline & Cerulenin $(\mu \mathrm{g} / \mathrm{ml})$ & $\mathrm{IC}_{30}(\mu \mathrm{M})$ & Sensitization factor $^{\mathrm{a}}$ & $\mathrm{IC}_{50}(\mu \mathrm{M})$ & Sensitization factor \\
\hline \multicolumn{6}{|c|}{ Simultaneous schedule } \\
\hline \multirow[t]{6}{*}{ 5-FU + Cerulenin } & 0 & $56.66 \pm 1.71$ & - & $78.84 \pm 2.77$ & - \\
\hline & 3 & $25.10 \pm 1.10$ & 2 & $49.76 \pm 0.77$ & 2 \\
\hline & 4 & $20.15 \pm 0.98$ & 3 & $33.01 \pm 3.62$ & 2 \\
\hline & 5 & $4.70 \pm 0.99$ & 12 & $8.35 \pm 0.88$ & 9 \\
\hline & 6 & $1.99 \pm 0.13$ & 28 & $3.8 \pm 0.47$ & 20 \\
\hline & 7 & N.A. & N.A. & N.A. & N.A. \\
\hline \multicolumn{6}{|c|}{ Sequential schedule I } \\
\hline \multirow[t]{6}{*}{ Cerulenin $\rightarrow 5$-FU } & 0 & N.A. & N.A. & $75,90 \pm 1.76$ & - \\
\hline & 3 & N.A. & N.A. & $40.50 \pm 0.31$ & 2 \\
\hline & 4 & N.A. & N.A. & $35.13 \pm 1.54$ & 2 \\
\hline & 5 & N.A. & N.A. & $20.36 \pm 0.29$ & 4 \\
\hline & 6 & N.A. & N.A. & $2.43 \pm 0.36$ & 31 \\
\hline & 7 & N.A. & N.A. & N.A. & N.A. \\
\hline \multicolumn{6}{|c|}{ Sequential schedule II } \\
\hline \multirow[t]{6}{*}{ 5-FU $\rightarrow$ Cerulenin } & 0 & N.A. & N.A. & $53.72 \pm 2.76$ & - \\
\hline & 3 & N.A. & N.A. & $18.27 \pm 0.88$ & 3 \\
\hline & 4 & N.A. & N.A. & $3.31 \pm 1.15$ & 16 \\
\hline & 5 & N.A. & N.A. & $0.83 \pm 0.15$ & 65 \\
\hline & 6 & N.A. & N.A. & $0.52 \pm 0.18$ & 107 \\
\hline & 7 & N.A. & N.A. & N.A. & N.A. \\
\hline
\end{tabular}

Data represent the cell viability effects upon exposure of SK-Br3 breast cancer cells to cerulenin and 5-FU in three different schedules of treatment. Briefly, $\mathrm{SK}-\mathrm{Br} 3$ cells were incubated in serial dilutions of 5-FU in the absence or presence of a given concentration of cerulenin. $\mathrm{IC}_{30}$ and $\mathrm{IC}_{50} \mathrm{values}$ are the concentrations of 5-FU which decreased cell viability by 30 and 50\%, respectively and measured by using the MTT assay as described in 'Materials and methods'. Values are mean of three independent experiments carried out in triplicate. 'Sensitization factors were obtained by dividing the $\mathrm{IC}_{30}$, ${ }_{50}$ values of 5 -FU as single agent by the $\mathrm{IC}_{30}, 50$ values obtained when cerulenin was supplemented. N.A. Not available (IC 30,50 values and sensitization factors are shown only where directly obtained from actual experiments).

expression of an isobole is $A_{\text {comb }} / A_{E}+B_{\text {comb }} / B_{E}=1$, with $A_{E}$ and $\mathrm{B}_{\mathrm{E}}$ the doses of $\mathrm{A}$ and $\mathrm{B}$ needed to produce the same effect $(\mathrm{E})$ and $A_{\text {comb }}$ and $B_{\text {comb }}$ the doses giving the same effect when combined. The resulting combination is considered as synergistic when $\mathrm{A}_{\text {comb }} / \mathrm{A}_{\mathrm{E}}+\mathrm{B}_{\text {comb }} / \mathrm{B}_{\mathrm{E}}<1$, antagonist when $\mathrm{A}_{\text {comb }} / \mathrm{A}_{\mathrm{E}}+\mathrm{B}_{\text {comb }} / \mathrm{B}_{\mathrm{E}}>1$ and additive when $\mathrm{A}_{\text {comb }} / \mathrm{A}_{\mathrm{E}}+\mathrm{B}_{\text {comb }} / \mathrm{B}_{\mathrm{E}}=1$. In a graphic representation of isoboles, a synergistic interaction is plotted below the line joining the doses that exert the same effect and an antagonist interaction is plotted above this line.

Statistical analysis. Statistical analysis was performed using the Student's t-test. Statistical significance levels were $\mathrm{P}<0.01$ and $\mathrm{P}<0.001$.

\section{Results}

Single agent activity of 5-FU and cerulenin in human breast cancer cell lines. To determine the cytotoxic activity of 5-FU and cerulenin as single agents, we first performed dose-effect experiments in a panel of human breast cancer cell lines naturally exhibiting graded amounts of FASN. FASN- and HER2-overexpressing SK-Br3 breast cancer cells were slightly less sensitive to the cytotoxic effects of 5-FU than MCF-7 and MDA-MB-231 cancer cells. Thus, the 5-FU concentrations needed for $50 \%$ growth inhibition (i.e., $\mathrm{IC}_{50}$ values) of lowFASN-expressing MDA-MB-231, moderate-FASN-expressing MCF-7 and FASN-overexpressing SK-BR3 breast cancer cells were $76 \pm 2,70 \pm 1$, and $79 \pm 3 \mu \mathrm{M}$, respectively. In agreement with our previous work (8-11), the extent of cerulenin-induced cytotoxicity significantly correlated with constitutive FASN levels in breast cancer cells, being the FASN-overexpressing SK-Br3 breast cancer cell line the most sensitive to FASN inhibition $\left(\mathrm{IC}_{50}=6.2 \pm 1 \mu \mathrm{g} / \mathrm{ml}\right.$ cerulenin versus $8.5 \pm 1$ and $9.1 \pm 1 \mu \mathrm{g} / \mathrm{ml}$ cerulenin in MCF-7 and MDA-MB-231 cells, respectively).

\section{Sensitizing effects of FASN inhibition on 5-FU-induced cytotoxicity}

Concurrent schedule $(5-F U+F A S N$ inhibition $24 \mathrm{~h})$. The effects of $24 \mathrm{~h}$ co-exposure to sub-optimal doses of cerulenin on the response of SK-Br3, MCF-7 and MDA-MB-231 breast cancer cells lines to 5-FU are shown in Tables I, II and III, respectively. To measure the increase in 5-FU sensitivity a 'sensitization factor' was determined by dividing 5-FU $\mathrm{IC}_{30},{ }_{50}$ values in the absence of cerulenin by those in the presence of increasing concentrations of the FASN inhibitor. Cerulenin co-exposure was found to enhance the cytotoxic activity of 5-FU in a dose-dependent manner in all the cell 
Table II. Effects of cerulenin-induced inhibition of FASN activity on the sensitivity of MCF-7 cells to 5-FU.

\begin{tabular}{|c|c|c|c|c|c|}
\hline & Cerulenin $(\mu \mathrm{g} / \mathrm{ml})$ & $\mathrm{IC}_{30}(\mu \mathrm{M})$ & Sensitization factor ${ }^{\mathrm{a}}$ & $\mathrm{IC}_{50}(\mu \mathrm{M})$ & Sensitization factor \\
\hline \multicolumn{6}{|c|}{ Simultaneous schedule } \\
\hline \multirow[t]{6}{*}{ 5-FU + Cerulenin } & 0 & N.A. & - & $70.13 \pm 0.86$ & - \\
\hline & 3 & N.A. & 2 & $28.23 \pm 0.32$ & 2 \\
\hline & 4 & N.A. & 3 & $20.12 \pm 0.85$ & 3 \\
\hline & 5 & N.A. & 7 & $10.23 \pm 0.38$ & 7 \\
\hline & 6 & N.A. & 81 & $0.86 \pm 0.10$ & 81 \\
\hline & 7 & N.A. & N.A. & N.A. & N.A. \\
\hline \multicolumn{6}{|c|}{ Sequential schedule I } \\
\hline \multirow[t]{6}{*}{ Cerulenin $\rightarrow 5$-FU } & 0 & $80.23 \pm 0.71$ & - & N.A. & N.A. \\
\hline & 3 & $40.41 \pm 0.92$ & 2 & N.A. & N.A. \\
\hline & 4 & $14.92 \pm 0.33$ & 5 & N.A. & N.A. \\
\hline & 5 & $12.22 \pm 0.44$ & 7 & N.A. & N.A. \\
\hline & 6 & $9.82 \pm 1.10$ & 8 & N.A. & N.A. \\
\hline & 7 & $0.92 \pm 0.33$ & 87 & N.A. & N.A. \\
\hline \multicolumn{6}{|c|}{ Sequential schedule II } \\
\hline \multirow[t]{6}{*}{ 5-FU $\rightarrow$ Cerulenin } & 0 & $4.55 \pm 0.21$ & - & $10.21 \pm 1.12$ & - \\
\hline & 3 & $0.63 \pm 0.12$ & 7 & $1.82 \pm 0.44$ & 6 \\
\hline & 4 & $0.45 \pm 0.21$ & 10 & $1.23 \pm 0.49$ & 8 \\
\hline & 5 & $0.35 \pm 0.22$ & 13 & $1.12 \pm 0.25$ & 9 \\
\hline & 6 & $0.25 \pm 0.16$ & 18 & $0.81 \pm 0.18$ & 12 \\
\hline & 7 & N.A. & N.A. & $0.51 \pm 0.21$ & 20 \\
\hline
\end{tabular}

Data represent the cell viability effects upon exposure of $\mathrm{MCF}-7$ breast cancer cells to cerulenin and 5-FU in three different schedules of treatment. Briefly, MCF-7 cells were incubated in serial dilutions of 5-FU in the absence or presence of a given concentration of cerulenin. $\mathrm{IC}_{30}$ and $\mathrm{IC}_{50}$ values are the concentrations of 5-FU which decreased cell viability by 30 and 50\%, respectively, and measured by using the MTT assay as described in 'Materials and

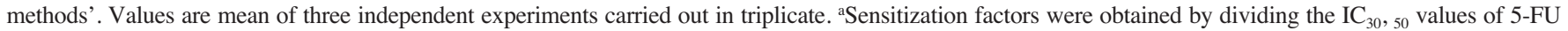
as single agent by the $\mathrm{IC}_{30},{ }_{50}$ values obtained when cerulenin was supplemented. N.A. Not available ( $\mathrm{IC}_{30},{ }_{50}$ values and sensitization factors are shown only where directly obtained from actual experiments).

lines tested. Thus, SK-Br3 cells incubated in the presence of cerulenin showed an up to 20 -fold increase $\mathrm{IC}_{50}$ in 5 -FU sensitivity (Table I). For MCF-7 cells, the addition of cerulenin to 5-FU markedly increased 5-FU-induced cytotoxicity up to 81 -fold at $\mathrm{IC}_{50}$ (Table II). In MDA-MB-231 cells, simultaneous exposure to 5-FU and the FASN inhibitor cerulenin enhanced 5 -FU cytotoxicity up to 58 -fold at $\mathrm{IC}_{50}$ (Table III).

Sequential schedule I (FASN inhibition $24 h \rightarrow 5-F U 24 h$ ). Pre-treatment with cerulenin $(24 \mathrm{~h})$ followed by the addition of 5-FU enhanced up to 31-fold the tumoricidal activity of 5-FU in SK-Br3 cells (Table I). A greater enhancement was observed in MCF-7 and MDA-MB-231 breast cancer cell lines (up to 87-fold Table II and 126-fold Table III at $\mathrm{IC}_{50}$, respectively).

Sequential schedule II (5-FU $24 h \rightarrow$ FASN inhibition). Pretreatment with 5 -FU followed by the addition of the FASN inhibitor cerulenin increased up to 107 -fold 5-FU cytotoxicity in SK-Br3 cells (Table I). Upon this schedule, a lower enhancement of 5-FU efficacy was observed in MCF-7 and MDA-MB-231 cells (up to 20-fold Table II and 18-fold Table III, respectively).
Nature of the interaction between the FASN blocker cerulenin and 5-FU. Cerulenin alone significantly decreased cell viability of human breast cancer cells at many of the concentrations examined. This indicated the presence of a potentially significant additive/antagonist component. Thus, possible synergistic interactions between cerulenin and 5-FU could not be accurately discriminated from additive or antagonistic effects on the basis of the above data. To detect in vitro synergy between these drug combinations, we carried out a series of isobologram transformations of multiple doseresponse analyses (12). Representative transformations are presented graphically (isobolograms) in Fig. 1. The dashed line drawn between the $\mathrm{IC}_{30}$, 50 values for cerulenin alone and the $\mathrm{IC}_{30},{ }_{50}$ values for 5-FU alone indicates the alignment of theoretical isoeffect data points for additive interactions between cerulenin and 5-FU. The true $\mathrm{IC}_{30},{ }_{50}$ points (the experimental concentrations of cerulenin and 5-FU which, when combined produced a 30 and $50 \%$ reduction of cell survival, respectively) were plotted and compared to the additive model of interaction. Data points above the dashed diagonal line of the additive effects in the isobole suggest antagonism and those below the diagonal suggest synergism.

Although isobolograms provide a graphical representation of cerulenin-5-FU interactions, the values of the mean 
Table III. Effects of cerulenin-induced inhibition of FASN activity on the sensitivity of MDA-MB-231 cells to 5-FU.

\begin{tabular}{|c|c|c|c|c|c|}
\hline & Cerulenin $(\mu \mathrm{g} / \mathrm{ml})$ & $\mathrm{IC}_{30}(\mu \mathrm{M})$ & Sensitization factor ${ }^{\mathrm{a}}$ & $\mathrm{IC}_{50}(\mu \mathrm{M})$ & Sensitization factor \\
\hline \multicolumn{6}{|c|}{ Simultaneous schedule } \\
\hline \multirow[t]{6}{*}{ 5-FU + Cerulenin } & 0 & N.A. & N.A. & $75.90 \pm 0.62$ & - \\
\hline & 3 & N.A. & N.A. & $37.99 \pm 0.95$ & 2 \\
\hline & 4 & N.A. & N.A. & $19.05 \pm 0.63$ & 4 \\
\hline & 5 & N.A. & N.A. & $11.89 \pm 0.52$ & 6 \\
\hline & 6 & N.A. & N.A. & $1.30 \pm 0.13$ & 58 \\
\hline & 7 & N.A. & N.A. & N.A. & N.A. \\
\hline \multicolumn{6}{|c|}{ Sequential schedule I } \\
\hline \multirow[t]{6}{*}{ Cerulenin $\rightarrow 5$-FU } & 0 & $83.4 \pm 1.26$ & - & N.A. & N.A. \\
\hline & 3 & $8.88 \pm 0.45$ & 9 & N.A. & N.A. \\
\hline & 4 & $5.19 \pm 0.37$ & 16 & N.A. & N.A. \\
\hline & 5 & $1.17 \pm 0.08$ & 71 & N.A. & N.A. \\
\hline & 6 & $0.66 \pm 0.08$ & 126 & N.A. & N.A. \\
\hline & 7 & N.A. & N.A. & N.A. & N.A. \\
\hline \multicolumn{6}{|c|}{ Sequential schedule II } \\
\hline \multirow[t]{6}{*}{ 5-FU $\rightarrow$ Cerulenin } & 0 & N.A. & N.A. & $16.01 \pm 0.78$ & - \\
\hline & 3 & N.A. & N.A. & $8.55 \pm 0.15$ & 2 \\
\hline & 4 & N.A. & N.A. & $3.97 \pm 0.52$ & 4 \\
\hline & 5 & N.A. & N.A. & $1.16 \pm 0.09$ & 14 \\
\hline & 6 & N.A. & N.A. & $0.90 \pm 0.05$ & 18 \\
\hline & 7 & N.A. & N.A. & N.A. & N.A. \\
\hline
\end{tabular}

Data represent the cell viability effects upon exposure of MDA-MB-231 breast cancer cells to cerulenin and 5-FU in three different schedules of treatment. Briefly, MDA-MB-231 cells were incubated in serial dilutions of 5-FU in the absence or presence of a given concentration of cerulenin. $\mathrm{IC}_{30}$ and $\mathrm{IC}_{50}$ values are the concentrations of 5-FU which decreased cell viability by 30 and 50\%, respectively and measured by using the MTT assay as described in 'Materials and methods'. Values are mean of three independent experiments carried out in triplicate. aSensitization factors were obtained by dividing the $\mathrm{IC}_{30},{ }_{50}$ values of 5 -FU as a single agent by the $\mathrm{IC}_{30}, 50$ values obtained when cerulenin was supplemented. N.A. Not available $\left(\mathrm{IC}_{30},{ }_{50}\right.$ values and sensitization factors are shown only where directly obtained from actual experiments).

Table IV. Combination Index $\left(\mathrm{CI}_{\mathrm{x}}\right)$ mean values of the interaction between 5-FU and cerulenin in human breast cancer cells.

\begin{tabular}{lcccccc}
\hline & \multicolumn{2}{c}{ 5-FU + Cerulenin } & \multicolumn{2}{c}{ 5-FU $\rightarrow$ Cerulenin } \\
Combination index at: & $\mathrm{CI}_{50}$ & \multicolumn{2}{c}{$\begin{array}{c}\text { Combination index at: } \\
\mathrm{CI}_{30}\end{array}$} & $\mathrm{CI}_{50}$ & \multicolumn{2}{c}{$\begin{array}{c}\text { Cerulenin } \rightarrow \text { 5-FU } \\
\text { Combination index at: } \\
\mathrm{CI}_{30}\end{array}$} \\
\hline MCF-7 & N.A. & $0.773 \pm 0.070^{\mathrm{a}}$ & $0.513 \pm 0.150^{\mathrm{b}}$ & $0.530 \pm 0.163^{\mathrm{b}}$ & $0.824 \pm 0.087^{\mathrm{a}}$ & N.A. \\
MDA-MB-231 & N.A. & $0.823 \pm 0.051^{\mathrm{a}}$ & N.A. & $0.808 \pm 0.140^{\mathrm{a}}$ & $0.656 \pm 0.131^{\mathrm{b}}$ & N.A. \\
SK-Br3 & $0.734 \pm 0.083^{\mathrm{a}}$ & $0.944 \pm 0.104$ & N.A. & $0.806 \pm 0.151^{\mathrm{a}}$ & N.A. & $1.013 \pm 0.074$ \\
\hline
\end{tabular}

Data represent the value of the mean $\mathrm{IC}_{30}$, 50 values of the interaction of cerulenin and 5-FU on SK-Br3, MCF-7 and MDA-MB-231 breast cancer cells. Student $\mathrm{t}$-tests were applied to formally evaluate whether synergism or antagonism was evident as compared to a null-hypothesized $\mathrm{CI}_{30},{ }_{50}$ of 1.0 ; ${ }^{\mathrm{a}} \mathrm{P}<01,{ }^{\mathrm{b}} \mathrm{P}<001$. N.A. Not available (CI indexes are shown only where directly obtained from actual experiments).

interaction index $\left(I_{x}\right)$ for a particular cell line, drug combination and effect levels are also labelled. In addition, a Student t-test was computed to evaluate whether significant differences in the $I_{x}$ means values occurred as compared to a null hypothesized $I_{x}$ of 1 (addition) and to formally evaluate whether antagonism or synergism was evident (Table IV). Upon concurrent administration of 5-FU and cerulenin, a moderate synergism occurred in all the cell lines tested. Prior cerulenin followed by the addition of 5-FU demonstrated additive interactions in SK-Br3 cells, whereas moderate to strong synergism was observed in MDA-MB-231 and MCF-7 cells, respectively. Pre-treatment with 5-FU followed by the addition of cerulenin promoted strong synergism in MCF-7 cells, while a moderate one occurred in MDA-MB-231 and SK-Br3 cells.

\section{Discussion}

The differential expression of FASN between cancer and normal cells may provide a valuable drug target for the 
MCF-7
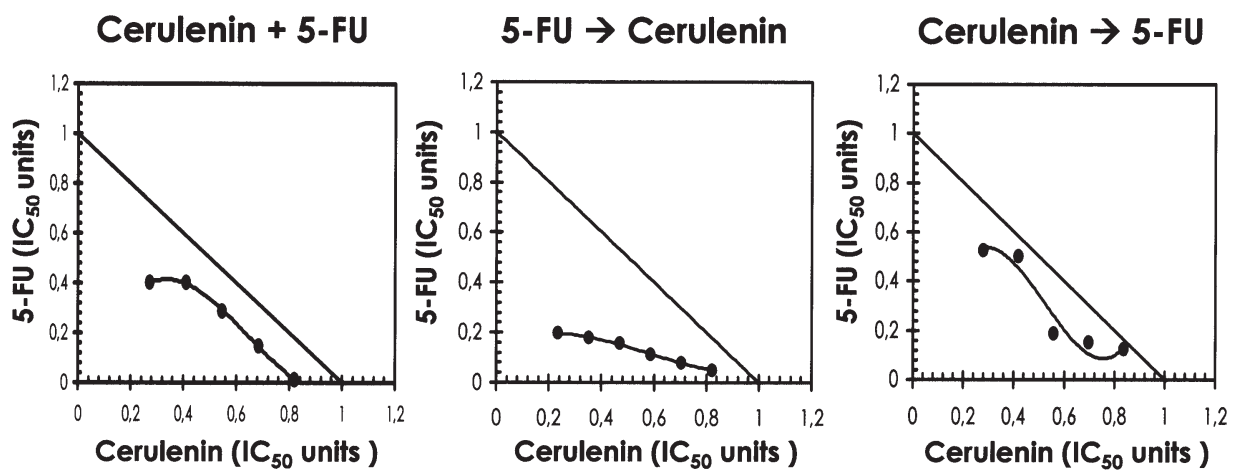

\section{MDA-MB-231}

Cerulenin + 5-FU

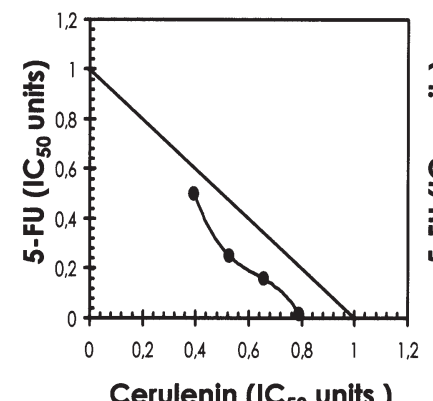

Cerulenin ( $\left(\mathrm{C}_{50}\right.$ units )
5-FU $\rightarrow$ Cerulenin

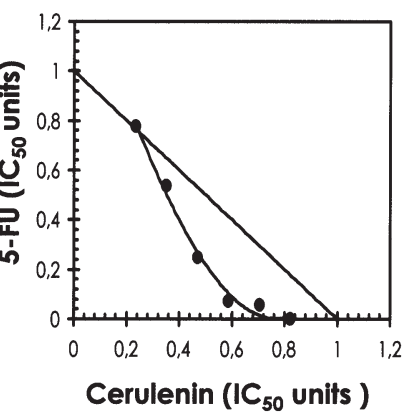

SK-Br3

5 -FU $\rightarrow$ Cerulenin

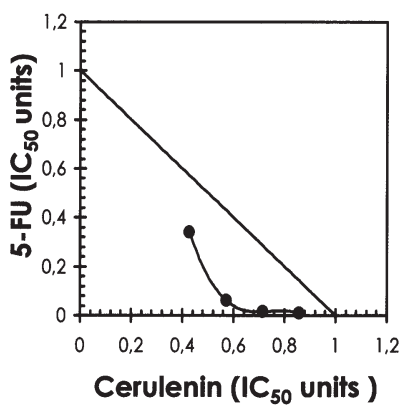

Cerulenin $\rightarrow$ 5-FU

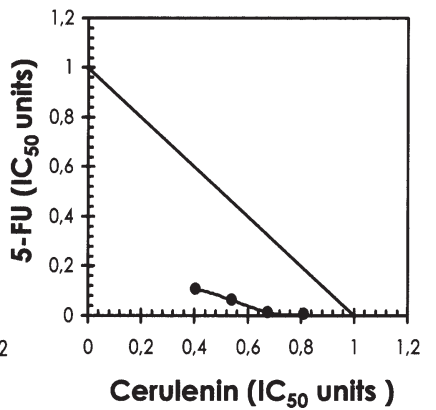

Cerulenin + 5-FU

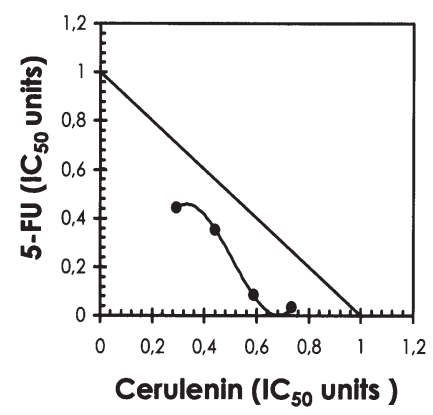

Cerulenin $\rightarrow 5$-FU

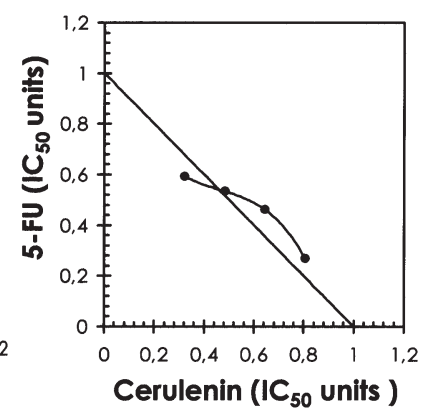

Figure 1. Synergy analyses of the interaction between cerulenin and 5-FU in human breast carcinoma cells. The nature of the interaction between cerulenin and 5-FU was evaluated by the isobologram technique, a dose-oriented geometric method of assessing drug interactions. Upon this approach, the concentration of cerulenin producing a desired (e.g., 30 or $50 \%$ inhibitory) effect was plotted on the horizontal axis and the concentration of 5-FU producing the same cytostatic degree was plotted on the vertical axis; a straight line joining these two points represents zero interaction between the two agents $\left(\mathrm{CI}_{30},{ }_{50}=1.0\right.$, additivity). An experimental isoeffect point is the concentration (expressed relative to cerulenin and 5-FU $\mathrm{IC}_{30}$ concentrations) of the two agents which when combined reduced cell viability by either 30 or $50 \%$. Within the designed assay range, a set of isoeffect points was generated because there were multiple cerulenin and 5-FU concentrations that achieved the same isoeffect. In our present study, the mean values of the survival fractions were used to generate the set of experimental isoeffect points and construct the isobole for a given cerulenin-5-FU combination. Data points above the diagonal line of the additive effects in the isobole suggest antagonism and those below the diagonal suggest synergism. Isobologram analysis was assessed only when obtained directly from actual experiments.

development of novel therapeutic anti-metabolites. Moreover, given the widespread expression of FASN in many types of human cancers (5), combinations of conventional agents with novel compounds directed against FASN-dependent endogenous fatty acid biosynthesis may result in increased efficacy over existing therapy for common human malignancies. Our current findings provide the first evidence of the synergistic nature in the cytotoxic interactions occurring when combining a chemical FASN inhibitor (i.e., cerulenin) and the anti-metabolite 5-FU in human breast cancer cells.

Our study reveals that pharmacological inhibition of FASN activity significantly enhances the cytotoxic effects of 5-FU 
toward human breast cancer cells (up to 126 times when MDA-MB-231 cells were exposed to cerulenin prior to 5-FU). To evaluate the nature of the combination, which may be synergistic, additive or antagonistic, we used the isobologram analysis, a mathematical method employed for assessing the combined effect of anti-tumoral drugs in vitro as a preclinical screening test (12). These analyses concluded that the nature of the interaction between the FASN inhibitor cerulenin and 5-FU in individual breast cancer cell lines generally exhibits sequence-dependency. Thus, at the cytotoxic condition $\left(\mathrm{IC}_{50}\right)$, synergism can mainly be observed when breast cancer cells are exposed to 5-FU prior to cerulenin whereas moderate synergism or additive interactions can be observed either when the chemical FASN blocker precedes 5-FU or when both drugs are concurrently administered. Of note, no antagonist interactions occur upon any schedule of combined treatment with cerulenin and 5-FU. From a clinical perspective, these findings strongly suggest that the administration of 5-FU prior to FASN inhibitors may be the optimal schedule for the combination in terms of cytotoxic effects in breast cancer.

Although the ultimate molecular mechanisms operating with these schedules are not addressed by our experiments, it can be suggested that various mechanisms by which FASN blockade leads to an enhanced 5-FU efficacy in breast cancer cells: a) Cerulenin-induced FASN blockade inhibits phospholipid synthesis indirectly by reducing the intracellular pool of diacylglycerides, the direct precursors for phospholipid synthesis (13). Most of the cellular accumulation of phospholipids in cycling cells in preparation for mitosis takes place during the S-phase (14). In this sense, cells in the Sphase are expected to be most sensitive to changes in phospholipid metabolism. Thus, a molecular mechanism underlying the synergism occurring when pre-treating a cell with 5-FU prior to cerulenin might relate to the ability of 5-FU to promote the accumulation of cells in early S-phase, making them more sensitive to the cytotoxic effects of cerulenin-induced FASN inhibition. b) Cancer cells have an unusual tolerance to limiting $\mathrm{O}_{2}$ availability, with an atypical carbohydrate metabolism displaying high rates of anaerobic glycolysis which provides precursors for FAs and DNA synthesis. In addition, some studies have found that cancer cells exhibiting an increased resistance to chemotherapeutic agents are characterized by displaying a higher use of fat, cytosolic glycolysis and when stressed, a reduced DNA damage (15). It has also been suggested that a novel approach to enhance tumor cell sensitivity to chemotherapy-induced cell death would favourably tailor the cellular redox status (16). Interestingly, the activation of FASN in cancer cells appears to represent a necessary survival strategy that occurs to compensate for an insufficiency of oxygen, providing an oxidizing power for key oxidative steps, thus improving the redox balance despite the surrounding conditions of extreme hypoxia (17-21). Pharmacological inhibition of FASN activity may change the whole metabolic status of breast cancer cells, thus modulating their responses to extracellular stimuli perturbing redox balance, including the chemotherapeutic agent 5-FU. c) Upon cell entry the antimetabolite 5-FU is converted to its active form 5-fluoro-2'-deoxyuridine monophosphate (FdUMP). In the presence of a reduced folate cofactor FdUMP forms a stable complex with thymidylate synthase with resultant inhibition of DNA synthesis. In this regard, 5-FU can be viewed as a pure S-phase-active chemotherapeutic agent (22). It has been reported that FASN inhibitors produce rapid, profound inhibition of DNA replication and S-phase progression in human cancer cells (23). Moreover, it has been recently reported that ceruleninmediated apoptosis is involved in adenine metabolic biosynthetic pathway (24). Thus, pre-treatment of cells with the FASN inhibitor cerulenin would lead to DNA synthesis inhibition in cancer cells, blocking 5-FU incorporation to DNA during replication and therefore making immediate 5-FU treatment less effective. d) It has been reported that certain specific proteins involved in glycolysis, FA synthesis (including FASN) and detoxification pathways are more highly expressed in HER2/neu-positive breast tumours when compared with HER2/neu-negative breast tumours (25). In addition, HER2 overexpression is associated with an increased resistance to multiple chemotherapeutic agents including MIAs, cisplatin or 5-FU (26-28). Considering our earlier reports revealing that FASN inhibition specifically suppresses HER 2/neu overexpression in cancer cells $(8,11)$, the synergistic interactions occurring between 5-FU and cerulenin in FASN- and HER2/neu-overexpressing breast cancer cells can be explained, at least in part, through FASN inhibitionrelated depletion of HER2/neu. In this sense, our current results support the notion that HER2/neu oncogene may act as the key molecular sensor of metabolism imbalance after the perturbation of tumor-associated FASN hyperactivity in breast cancer cells (29).

In summary, we report the observation that the pharmacological blockade of tumour-associated FASN hyperactivity can synergistically sensitize breast cancer cells to 5-FUinduced cell killing. Considering that FASN activity has previously been involved in the efficacy of MIAs in breast cancer cells, it is fair to suggest that novel therapeutic strategies aimed at suppressing breast cancer-associated FASN hyperactivity can be exploited to improve the efficacy of existing breast cancer chemotherapies (30).

\section{Acknowledgements}

Javier A. Menendez is the recipient of a Basic, Clinical and Translational Research Award (BCTR0600894) from the Susan G. Komen Breast Cancer Foundation (Texas, USA). This work was also supported by the Instituto de Salud Carlos III (Ministerio de Sanidad y Consumo, Fondo de Investigación Sanitaria - FIS-, Spain; grants CP05-00090, PI06-0778 to Javier A. Menendez, and RD06-0020-0028 to Ramon Colomer, Joan Brunet and Javier A. Menendez).

\section{References}

1. Weiss L, Hoffmann GE, Schreiber R, Andres H, Fuchs E, Korber E and Kolb HJ: Fatty-acid biosynthesis in man, a pathway of minor importance. Purification, optimal assay conditions and organ distribution of fatty-acid synthase. Biol Chem Hoppe Seyler 367: 905-912, 1986.

2. Sul HS and Wang D: Nutritional and hormonal regulation of enzymes in fat synthesis: studies of fatty acid synthase and mitochondrial glycerol-3-phosphate acyltransferase gene transcription. Annu Rev Nutr 18: 331-351, 1998.

3. Menendez JA and Lupu R: Fatty acid synthase-catalyzed de novo fatty acid biosynthesis: from anabolic-energy-storage pathway in normal tissues to jack-of-all-trades in cancer cells. Arch Immunol Ther Exp 52: 414-426, 2004. 
4. Sul HS, Smas CM, Wang D and Chen L: Regulation of fat synthesis and adipose differentiation. Prog Nucleic Acid Res Mol Biol 60: 317-345, 1998.

5. Kuhajda FP: Fatty-acid synthase and human cancer: new perspectives on its role in tumor biology. Nutrition 16: 202-208, 2000.

6. Kuhajda FP, Jenner K, Wood FD, Hennigar RA, Jacobs LB, Dick JD and Pasternack GR: Fatty acid synthesis: a potential selective target for antineoplastic therapy. Proc Natl Acad Sci USA 91: 6379-6383, 1994.

7. Longley DB, Harkin DP and Johnston PG: 5-fluorouracil: mechanisms of action and clinical strategies. Nat Rev Cancer 3: 330-338, 2003.

8. Menendez JA, Lupu R and Colomer R: Inhibition of tumorassociated fatty acid synthase hyperactivity induces synergistic chemosensitization of HER-2/neu-overexpressing human breast cancer cells to docetaxel (taxotere). Breast Cancer Res Treat 84: 183-195, 2004.

9. Menendez JA, Vellon L, Colomer R and Lupu R: Pharmacological and small interference RNA-mediated inhibition of breast cancer-associated fatty acid synthase (oncogenic antigen-519) synergistically enhances Taxol (paclitaxel)-induced cytotoxicity. Int J Cancer 115: 19-35, 2005.

10. Menendez JA, Colomer R and Lupu R: Inhibition of tumorassociated fatty acid synthase activity enhances vinorelbine (Navelbine)-induced cytotoxicity and apoptotic cell death in human breast cancer cells. Oncol Rep 12: 411-422, 2004.

11. Menendez JA, Vellon L, Mehmi I, Oza BP, Ropero S, Colomer R and Lupu R: Inhibition of fatty acid synthase (FAS) suppresses HER2/neu (erbB-2) oncogene overexpression in cancer cells. Proc Natl Acad Sci USA 101: 10715-10720, 2004.

12. Berenbaum MC: What is synergy? Pharmacol Rev 41: 93-141, 1989.

13. Jackowski S, Wang $\mathrm{J}$ and Baburina I: Activity of the phosphatidylcholine biosynthetic pathway modulates the distribution of fatty acids into glycerolipids in proliferating cells. Biochim Biophys Acta 1483: 301-315, 2000.

14. Jackowski S: Coordination of membrane phospholipid synthesis with the cell cycle. J Biol Chem 269: 3858-3867, 1994.

15. Harper ME, Antoniou A, Villalobos-Menuey E, Russo A, Trauger R, Vendemelio M, George A, Bartholomew R, Carlo D, Shaikh A, Kupperman J, Newell EW, Bespalov IA, Wallace SS, Liu Y, Rogers JR, Gibbs GL, Leahy JL, Camley RE, Melamede R and Newell MK: Characterization of a novel metabolic strategy used by drug-resistant tumor cells. FASEB J 16: 1550-1557, 2002.

16. Pervaiz S and Clement MV: Tumor intracellular redox status and drug resistance-serendipity or a causal relationship? Curr Pharm Des 10: 1969-1977, 2004.
17. Hochachka PW, Rupert JL, Goldenberg L, Gleave M and Kozlowski P: Going malignant: the hypoxia-cancer connection in the prostate. Bioessays 24: 749-757, 2002.

18. Baron A, Migita T, Tang D and Loda M: Fatty acid synthase: a metabolic oncogene in prostate cancer? J Cell Biochem 91: 47-53, 2004

19. Menendez JA, Colomer R and Lupu R: Why does tumorassociated fatty acid synthase (oncogenic antigen-519) ignore dietary fatty acids? Med Hypotheses 64: 342-349, 2005.

20. Menendez JA and Lupu R: Oncogenic properties of the endogenous fatty acid metabolism: molecular pathology of fatty acid synthase in cancer cells. Curr Opin Clin Nutr Metab Care 9: 346-357, 2006.

21. Brown JM and Giaccia AJ: The unique physiology of solid tumors: opportunities (and problems) for cancer therapy. Cancer Res 58: 1408-1416, 1998.

22. Shah MA and Schwartz GK: Cell cycle-mediated drug resistance: an emerging concept in cancer therapy. Clin Cancer Res 7: 2168-2181, 2001.

23. Pizer ES, Chrest FJ, DiGiuseppe JA and Han WF: Pharmacological inhibitors of mammalian fatty acid synthase suppress DNA replication and induce apoptosis in tumor cell lines. Cancer Res 58: 4611-4615, 1998.

24. Chung KS, Sun NK, Lee SH, Lee HJ, Choi SJ, Kim SK, Song JH, Jang YJ, Song KB, Yoo HS, Simon J and Won M: Ceruleninmediated apoptosis is involved in adenine metabolic pathway. Biochem Biophys Res Commun 349: 1025-1031, 2006.

25. Zhang D, Tai LK, Wong LL, Chiu LL, Sethi SK and Koay ES: Proteomic study reveals that proteins involved in metabolic and detoxification pathways are highly expressed in HER-2/neupositive breast cancer. Mol Cell Proteomics 4: 1686-1696, 2005.

26. Alauoi-Jamali MA, Paterson J, Al Moustafa AE and Yen L: The role of ErbB-2 tyrosine kinase receptor in cellular intrinsic chemoresistance: mechanisms and implications. Biochem Cell Biol 75: 315-325, 1997.

27. Kim R, Tanabe K, Uchida Y, Osaki A and Toge T: The role of HER-2 oncoprotein in drug-sensitivity in breast cancer (Review). Oncol Rep 9: 3-9, 2002.

28. Knuefermann C, Lu Y, Liu B, Jin W, Liang K, Wu L, Schmidt M, Mills GB, Mendelsohn J and Fan Z: HER2/PI-3K/Akt activation leads to a multidrug resistance in human breast adenocarcinoma cells. Oncogene 22: 3205-3212, 2003.

29. Menendez JA, Lupu R and Colomer R: Targeting fatty acid synthase: potential for therapeutic intervention in her-2/neuoverexpressing breast cancer. Drug News Perspect 18: 375-385, 2005.

30. Lupu R and Menendez JA: Pharmacological inhibitors of fatty acid synthase (FASN)-catalyzed endogenous fatty acid biogenesis: a new family of anti-cancer agents? Curr Pharm Biotechnol 7: 483-493, 2006. 